\title{
Klimarelevanz der Abfallwirtschaft aus Sicht eines Sammlers
}

\section{Climatological relevance of waste management as seen by a collector}

\author{
von R. Pomberger, A. Curtis, T. Scherübl
}

\section{Kurzfassung/Summary}

Die Abfallwirtschaft spielt bei der Erreichung der $\mathrm{CO}_{2}$-Reduktionsziele in Österreich eine wichtige Rolle, sie ist einer der wenigen Sektoren die eine positive Entwicklung darstellen können. Die Umsetzung der Deponie-VO hat zu wesentlichen strukturellen Veränderungen in der Entsorgungswirtschaft geführt und insbesondere durch Vermeidung von Methanemissionen aus Deponien starke $\mathrm{CO}_{2}$-Reduktionen bewirkt. Die optimierte energetische Nutzung von Abfällen in Müllverbrennungsanlagen und industriellen Mitverbrennungsanlagen führt zur Verminderung bei Treibhausgasen. Der $\mathrm{CO}_{2}$-Beitrag von Logistik und Aufbereitung ist bei Ersatzbrennstoffen $\mathrm{zu}$ vernachlässigen. Besondere Bedeutung hat die getrennte Sammlung und stoffliche Verwertung, da dadurch wesentliche Treibhausgasgutschriften generiert werden können. Es werden unternehmensspezifische Beiträge zum Klimaschutz wie z. B. die Erstellung einer Energie- und Klimabilanz eines Entsorgungsunternehmens, die Auswirkungen von Logistikoptimierungen, der Einsatz emissionsarmer Fahrzeuge und der Ausbau der getrennten Sammlung in Zentral- und Osteuropa dargestellt. Die europäische Abfallwirtschaft bietet auch in $\mathrm{Zu}$ kunft wesentliche Treibhauseinsparungspotentiale, wenn der Standard der Abfallbehandlung angepasst wird.

Waste management is an important factor to be considered in the attempt to meet $\mathrm{CO}_{2}$ reduction goals in Austria, since this is one of the few sectors likely to show positive development. The implementation of the Austrian Landfill Law has brought about substantial changes in waste management and, by avoiding methane emissions from landfills, has substantially reduced $\mathrm{CO}_{2}$ emissions. The optimised energetic use of refuse in waste incineration plants and combined industrial incineration plants reduces greenhouse-gas emissions. The $\mathrm{CO}_{2}$ contribution involved in the logistics and processing of substitute fuels is negligible. Of particular importance is separated collection and material utilisation as this helps to generate important greenhouse-gas credits. The author describes individual companies' contributions to climate protection, such as an energy and climate balance drawn up by a waste-disposal company, logistics optimisations and their effects, the use of low-emission vehicles and the development of separated waste collection systems in Central and Eastern Europe. Waste management in Europe offers substantial greenhouse-gas saving potentials also for the future if the standard of waste treatment is upgraded accordingly.1. Einführung

\section{Einleitung}

Auf internationaler Ebene ist das Thema „Klimaschutz“ bereits seit ca. 20 Jahren als weltweit zu lösende Aufgabe definiert (Toronto 1988, Rio de Janeiro 1992, Kyoto 1997). Heute ist es ein gesellschaftlich und medial umfassend diskutiertes Thema und daraus abgeleitete Maßnahmen zur Reduktion von Treibhausgasen betreffen Wirt- schaft und BürgerInnen mehr oder weniger direkt.

Die „Strategie Österreichs zur Erreichung des Kyotozieles“ - kurz „Klimastrategie“ genannt (Bundesregierung 2002, [1]) - legte Maßnahmen und Zielwerte zur Reduktion von Treibhausgasen in 8 Sektoren fest. Insgesamt soll bis 2010 eine Gesamtreduktion von 9,1\% erreicht werden.

Die Abfallwirtschaft spielt bei der Erreichung der Reduktionsziele eine wichtige Rolle. Die Abfallwirtschaft ist einer der wenigen Sektoren, die eine sehr positive Entwicklung darstellen können. Interessant ist, dass in der Öffentlichkeit die überaus positiven Beiträge der Abfallwirtschaft nur geringes mediales Echo erreicht.

Unternehmen der Abfallwirtschaft sind direkt oder indirekt von Maßnahmen zur Erreichung der Klimaziele betroffen. Jedenfalls müssen sie sich mit den Risiken und Chancen von Maßnahmen zum Klimaschutz beschäftigen.

Der Begriff „Abfallsammler“ wird in dieser Arbeit nicht reduziert auf Unternehmen der Entsorgungswirtschaft, die ausschließlich Abfälle sammeln. Da „Abfallsammlung“ am Beginn der Dienstleistungskette steht, ist sie nicht von den nachfolgenden Prozessen der Abfallbehandlung zu trennen. Es obliegt ja vielfach dem Abfallsammler was er sammelt, wie viel er sammelt und welche nachfolgende Behandlung er entweder selbst (in eigenen Anlagen) durchführt oder an welche Behandler er die gesammelten Abfälle zur Verwertung oder Beseitigung weitergibt. Der Prozess der „Abfallsammlung“ sollte daher gemeinsam mit 


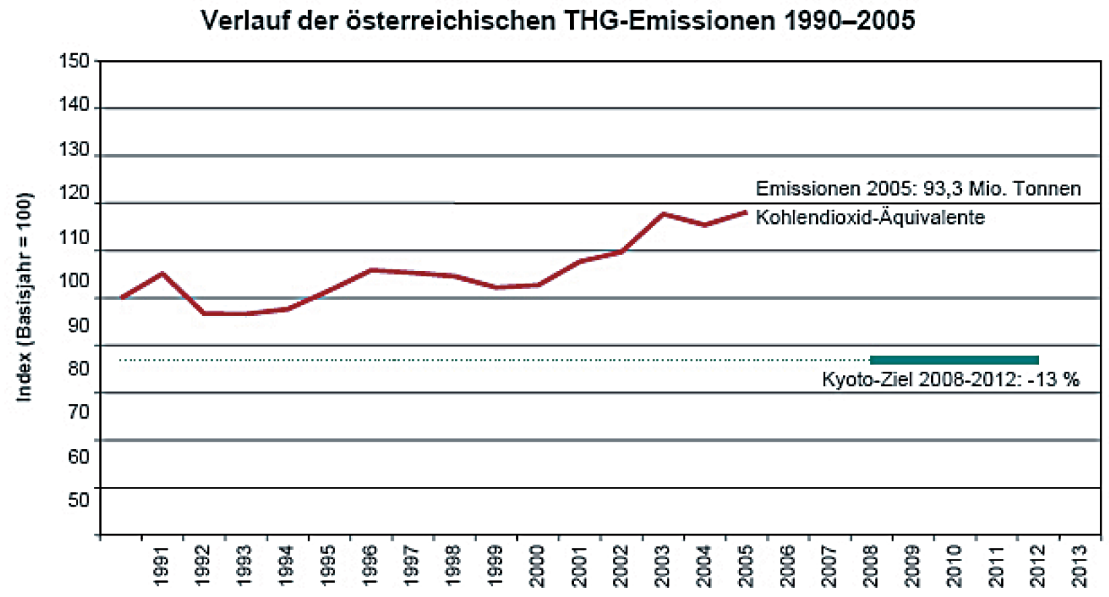

Abb. 1: Abweichung der tatsächlichen Treibhausgasemissionen Österreichs zum Kyotoziel [2]

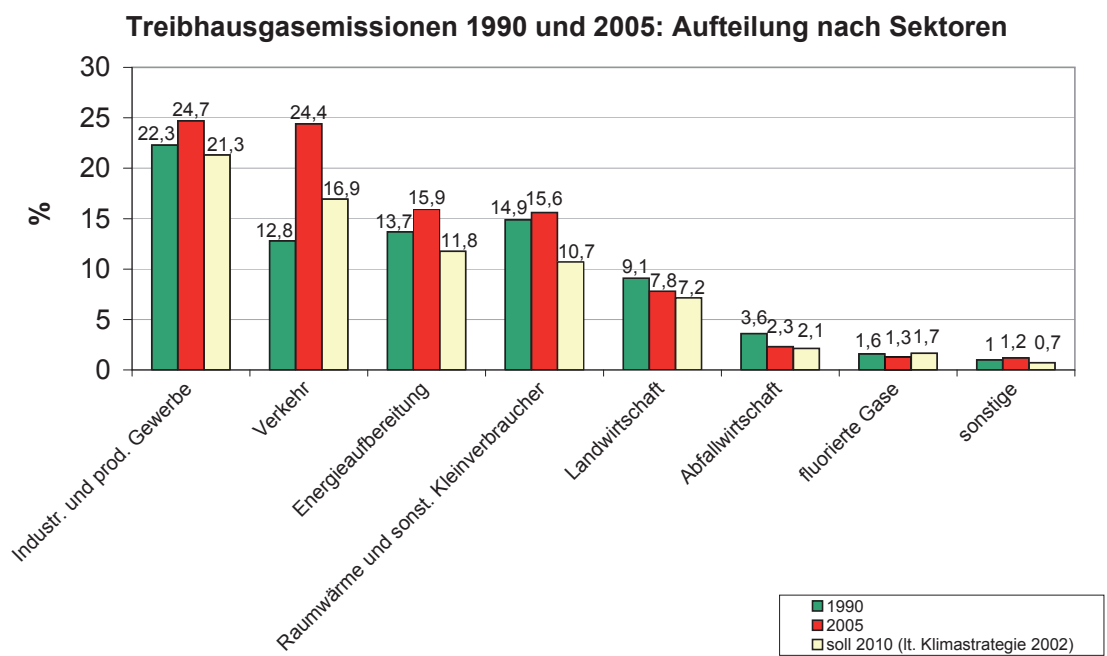

Abb. 2: Treibhausgasemissionen 1990 und 2005: Aufteilung nach Sektoren [1]

Die Entwicklung der Treibhausgasemissionen 1990-2005

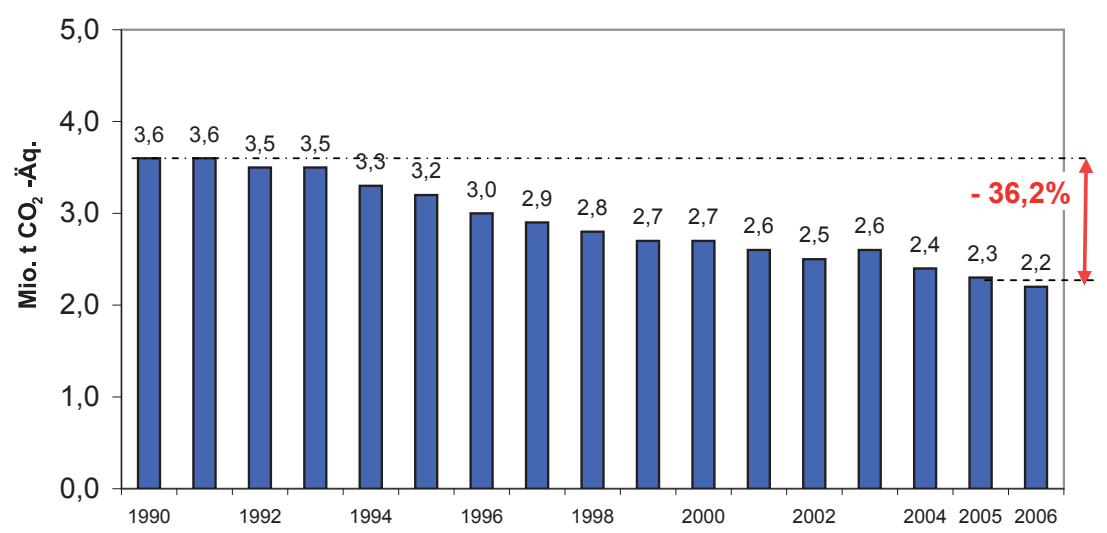

Abb. 3: Entwicklung der Treibhausgasemissionen 1990-2005 des Sektors Abfallwirtschaft [2] der nachfolgenden „Behandlung“ gesehen werden.

Die reale österreichische Entsorgungswirtschaft zeigt, dass insbesondere die größeren Entsorgungsunternehmen (private wie kommunale) ihre Wertschöpfungskette durch Behandlungsanlagen erweitert haben oder eng mit Verwertungs-/Behandlungsunternehmen kooperieren.

Abfallsammler sind daher vielfach auch selber Behandler/Verwerter oder üben zumindest hohen Einfluss auf die nachfolgende Behandlung von Abfällen aus. Abfallsammler i. S. dieser Arbeit ist daher umfassender als. „Abfallentsorgungsunternehmen" zu sehen.

Einige positive Treibhausgas reduzierende Maßnahmen, die Entsorgungsunternehmen setzen oder setzen könnten, werden aber nicht dem Sektor Abfallwirtschaft zugerechnet; z. B. wird eine Optimierung in der Logistik (weniger Transporte durch Belieferung regionaler Verwerter) dem Sektor Verkehr zugeordnet. Ähnliches gilt auch für andere Effekte abfallwirtschaftlicher Maßnahmen (Vorteile Ersatzbrennstoffe zu Industriesektor, Vorteile der Biogasanlagen zu Sektor Landwirtschaft).

\section{Klimaschutz - allgemein}

Das Kyotoziel sieht bis 2012 eine Treibhausgasreduktion von $8 \%$ für die EU vor (bezogen auf das Basisjahr 1990).

Der geplante Beitrag Österreichs beträgt aufgrund EU interner Regelungen eine höhere Reduktion von $13 \%$ (1990: 79,1 Mio. t/a Treibhausgase).

Bis 2005 konnte keine Reduktion der Emissionen erreicht werden, im Gegenteil die Emissionen stiegen um 14,2 Mio.t gegenüber dem Basisjahr 1990. Abbildung 1 zeigt, dass die Emissionen 2005 in Österreich rund 24,4 Mio.t über dem Kyotoziel lagen.

Hauptverantwortlich für die Ab- 
weichung ist die starke Zunahme von Treibhausgasemissionen des Sektors Verkehr. Diese Zunahme ist deutlich höher als die Reduktion in den Sektoren Industrie und produzierende Gewerbe, Landwirtschaft und Abfallwirtschaft. Abbildung 2 zeigt die Entwicklung der Treibhausgasemissionen in den einzelnen Sektoren. Abbildung 3 zeigt die positive Entwicklung des Sektors Abfallwirtschaft.

Den wichtigsten Beitrag des Abfallsektors lieferten die Maßnahmen zur verminderten Freisetzung von Methan aus Deponien; z. B. die verbesserte Erfassung und energetische Nutzung der Deponiegase und das seit 01.01.2004 geltende Deponierungsverbot für nicht vorbehandelte Abfälle (Deponie-Verordnung 1996 (DVO)). Allein das Auslaufen der Ausnahmeregelungen zur DVO lässt eine weitere deutliche Reduktion im Sektor Abfallwirtschaft erwarten.

Allerdings umfasst die Bilanz des Sektors Abfallwirtschaft nur die vermiedenen Emissionen aus Deponien; andere positive Maßnahmen der Abfallwirtschaft wie energetische Nutzung von Abfällen als Ersatzbrennstoffe oder verstärkte stoffliche Verwertung von Abfällen werden als Gutschrift nicht diesem Sektor zugeschlagen. In einer umfassenden Bewertung der Abfallwirtschaft in ihrem Beitrag zum Klimaschutz muss das aber berücksichtigt werden. Der positive Beitrag der Abfallwirtschaft geht daher über die in der Bilanz des Sektors Abfallwirtschaft (-36,2 \% 1990-2005 lt. Kyoto-Fortschrittsbericht) dargestellten Effekte deutlich hinaus.

\section{Beiträge der Abfallwirt- schaft zum Klimaschutz}

\subsection{Deponie-Verordnung (DVO)}

Die DVO hat zweifellos die österreichische Abfallwirtschaft maß- geblich beeinflusst. In Bezug auf den Klimaschutz zeigt sich, dass der wesentliche Effekt der DVO die weitgehende Vermeidung von $\mathrm{Me}$ thanemissionen aus Deponien ist.

Aus Sicht der Abfallentsorgungsunternehmen entscheidend war, dass der etablierte und kostengünstige Entsorgungsweg „Deponie" für wesentliche Abfallströme ab 2004 nicht mehr zur Verfügung stand.

Es mussten daher Alternativen zur Deponie gefunden und aufgebaut werden. Die gewählten Strategien waren unterschiedlich. Die Bandbreite reicht vom Bau eigener Müllverbrennungs-anlagen über Kooperationen mit industriellen Mitverbrennungsanlagen bis hin zu Abwarten und Wahrnehmen kurzfristiger Entsorgungslösungen von AbfallhändlernInnen.

Jede der Bewältigungsstrategien bietet Chancen und Risiken, wobei sich zwei Konzepte aus derzeitiger Sichtweise als besonders erfolgreich darstellen:

1. Ersatz der Deponie durch die klassische Müllverbrennungsanlagen mit hohem energetischem Wirkungsgrad (Strom und Wärme).

2. Kombination von mechanischen Vorbehandlungsanlagen (MBA, Splittinganlagen, Ersatzbrennstoff-Produktionsanlagen) und Kooperation mit industriellen Mitverbrennungsanlagen (als Ersatzbrennstoff-Verwerter).

Eine weitere Auswirkung der DVO sind verstärkte Aktivitäten der Entsorgungsunternehmen im
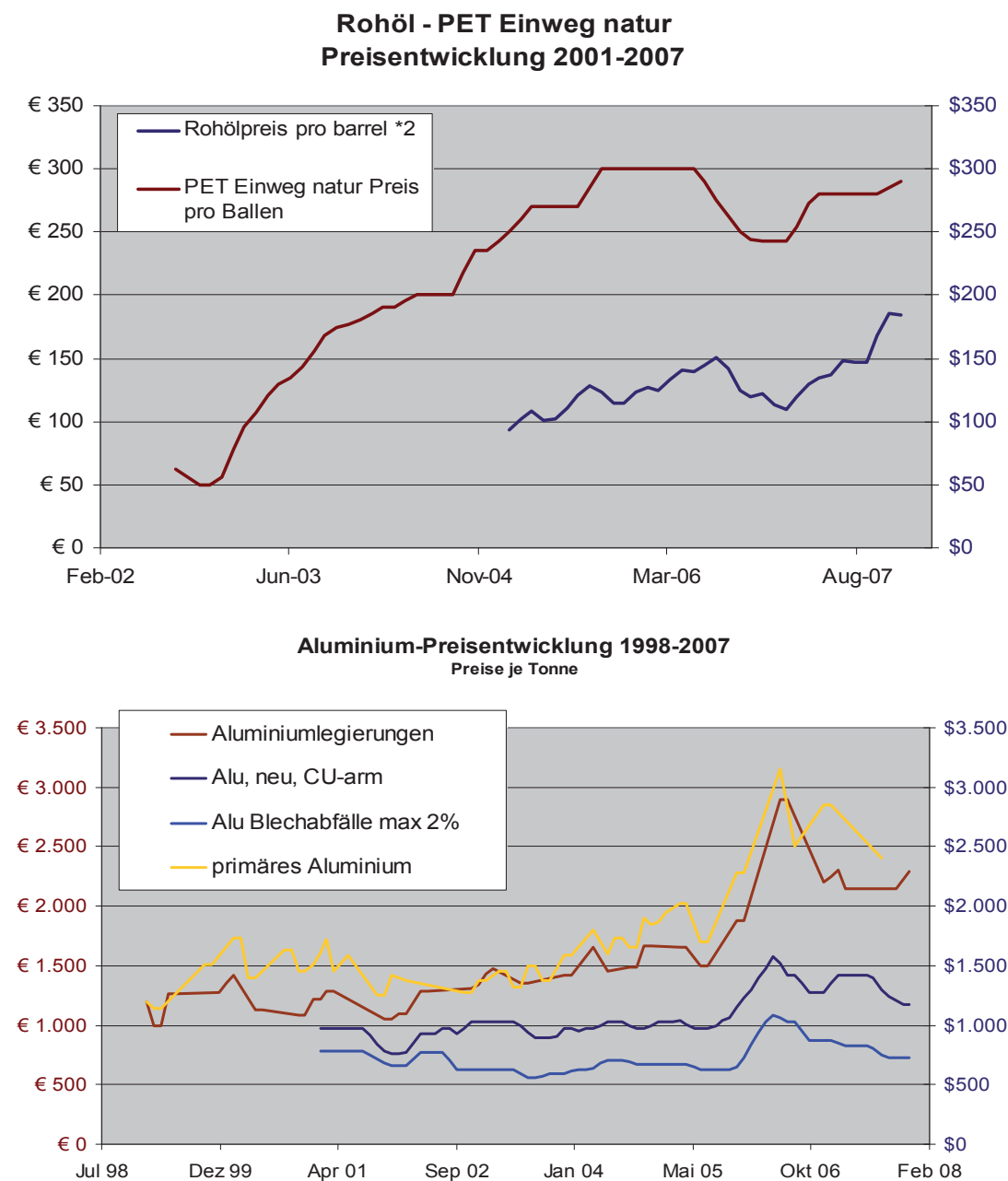

Abb. 4a und b: Preiskorrelationen Rohstoffe - Wertstoffe - Energie [3] 


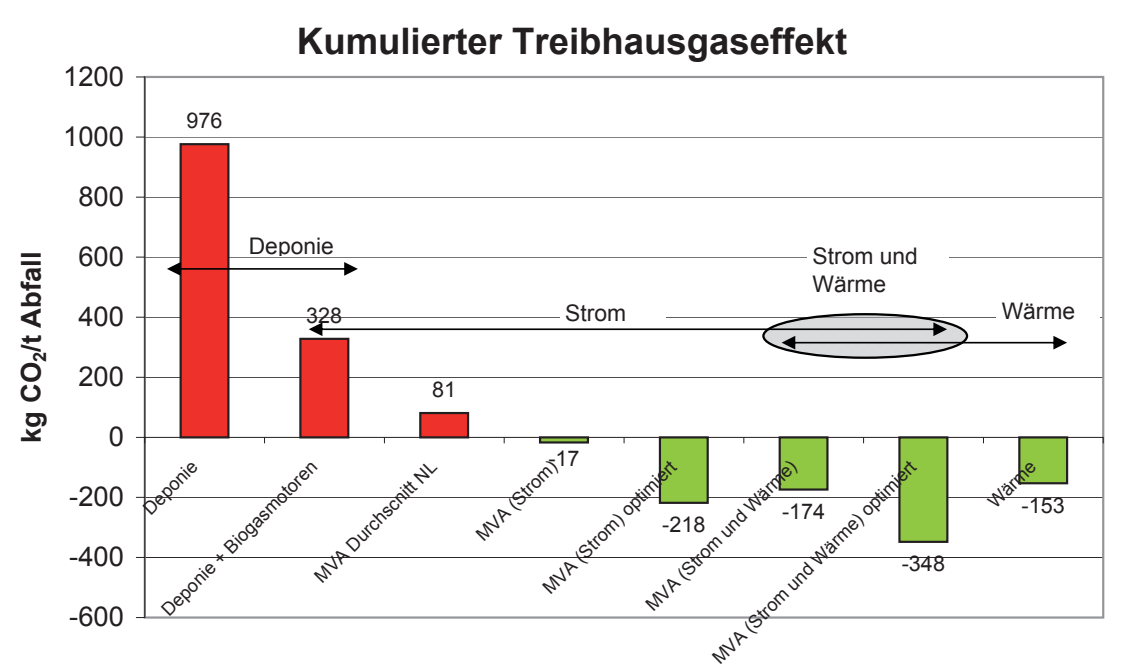

Abb. 5: Kumulierter Treibhauseffekt [4]

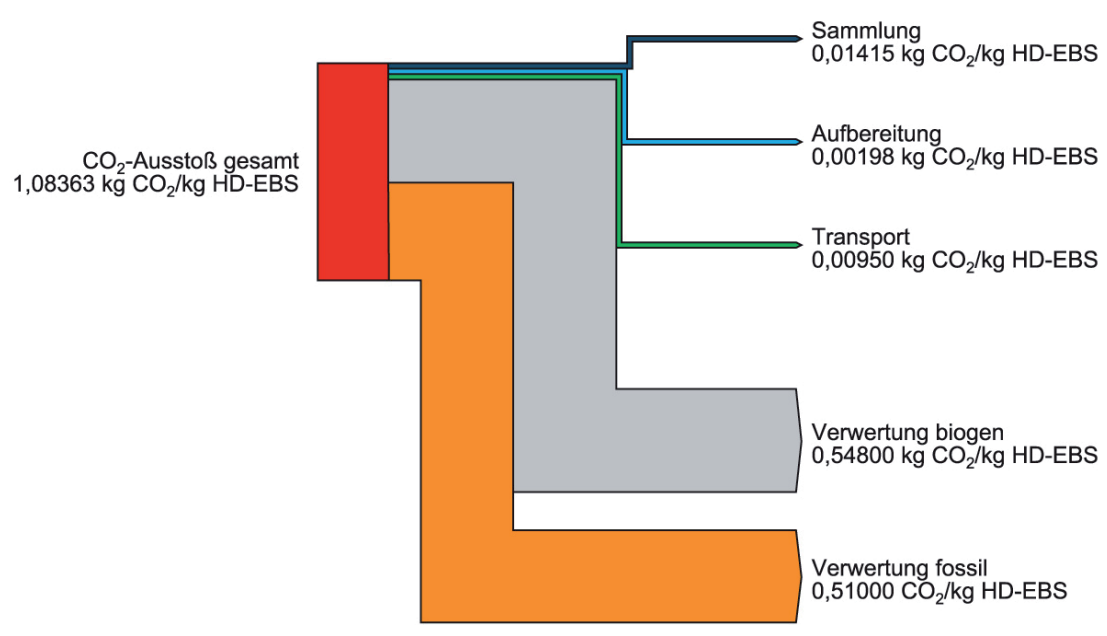

Abb. 6: Sankeydarstellung der $\mathrm{CO}_{2}$-Bilanz eines EBS-Einsatzes im Zementwerk

Bereich stoffliche Verwertung. Die Begrenzung der Verbrennungskapazitäten und steigende Preise bei thermischer Behandlung machten die getrennte Erfassung von Wertstoffen und die Sortierung - insbesondere von Abfällen aus dem Gewerbebereich - wieder attraktiv. Unterstützt wurde dieser Trend durch international stark gestiegene Rohstoff- und Energiepreise (siehe Abbildung 4), die das getrennte Sammeln und Aussortieren von verwertbaren Fraktionen ökonomisch bevorzugten.

Zusammenfassend hat sich die DVO nicht nur durch ihre direkten Effekte (Methanreduktion), son- dern auch durch ihre indirekten Effekte (Steigerung der Verwertung, Steigerung der energetischen Verwertung) als massiver Motor für positive klimarelevante Entwicklungen in der Abfallwirtschaft bewiesen.

\subsection{Energie in Müllverbren- nungsanlagen}

Energienutzung aus Abfällen kann einerseits in Müllverbrennungsanlagen und andererseits in industriellen Feuerungsanlagen (Mitverbrennungsanlagen) erfolgen.

Der positive Beitrag zum Klimaschutz ist die Differenz von di- rekt erzeugten $\mathrm{CO}_{2}$-Emissionen $\mathrm{CO}_{2}$ aus fossilem Kohlenstoff, $\mathrm{CO}_{2}$ aus biogenem Kohlenstoff, Methan-Emissionen) und vermiedenem $\mathrm{CO}_{2}$ (durch Elektrizitätsproduktion, durch Wärmeproduktion, durch Metallrecycling, durch $\mathrm{CO}_{2}$ aus biogenem Kohlenstoff). Aufgrund mehrere Aspekte stellt die Verbrennung von Siedlungsabfällen meist eine Verbesserung im Vergleich zur Deponierung dar:

- Die Methanproduktion auf Deponien wird vermieden.

- Der biogene Kohlenstoff in den Abfällen ist bei der Verbrennung klimaneutral.

- Die energetische Nutzung von Abfällen führt zur Einsparung von fossilen Rohstoffen (Substitution) und damit verbunden zur Reduktion von klimawirksamen $\mathrm{CO}_{2}$ Emissionen (abhängig vom Wirkungsgrad der Verbrennungsanlage)

Eine Life-Cycle-Analyse (LCA)Studie aus den Niederlanden [4] hat verschiedene Szenarien berechnet (siehe Abbildung 5). Die Studie weist für die Deponie (mit Deponiegasnutzung) eine Belastung von $328 \mathrm{~kg} \mathrm{CO}$ äq/t Abfall aus, ohne Deponiegasnutzung werden $976 \mathrm{~kg} \quad \mathrm{CO}_{2}$ äq/t Abfall emittiert.

Konventionelle Abfallverbrennungsanlagen (MVA) weisen demnach folgende $\mathrm{CO}_{2}$-Emissionen pro t Abfall auf: ( +Nettobelastung, -Nettoentlastung )

- Durchschnitt der MVA Niederlande (Stand 2005) +81 kg $\mathrm{CO}_{2}$ äq/t Abfall

- durchschnittliche MVA (Strom $20 \%$ WG) - $17 \mathrm{~kg} \mathrm{CO}_{2}$ äq/t Abfall

- durchschnittliche MVA (Strom + Wärme) -174 kg CO 2 äq/t Abfall

Optimierte Abfallverbrennungsanlagen (MVA) erreichen folgende $\mathrm{CO}_{2}$-Emissionen pro $\mathrm{t} \mathrm{Ab}$ fall:

- MVA Strom optimiert (30 \% WG) $-218 \mathrm{~kg} \mathrm{CO}_{2}$ äq/t Abfall 
- MVA Strom + Wärme optimiert $-348 \mathrm{~kg} \mathrm{CO} \mathrm{Ca}_{2}$ at Abfall

Die Ergebnisse dieser Studie sollen die Unterschiede verschiedener Konzepte und ihre Größenordnung in Bezug auf Treibhausgasemissionen zeigen. Konkrete österreichische Anlagen können aufgrund ihrer spezifischen Rahmenbedingungen abweichende Werte aufweisen.

Die in Abbildung 5 dargestellten Werte stimmen gut mit den Werten aus dem deutschen Umweltbundesamt überein [5]. Hier wird für deutsche Müllverbrennungsanlagen im Durchschnitt eine $\mathrm{CO}_{2}-$ Gutschrift in der Höhe von $184 \mathrm{~kg}$ $\mathrm{CO}_{2} / \mathrm{t}$ Abfall angegeben

Die Optimierung der Energieeffizienz in Müllverbrennungsanlagen, d.h. Abfallverbrennung wird als Energieerzeugungsprozess gesehen und geführt, kann die Gutschrift für die Abfallverbrennung auf $348 \mathrm{~kg} \mathrm{CO}_{2} / \mathrm{t}$ Abfall [4] bzw. $333 \mathrm{~kg} \mathrm{CO}_{2} / \mathrm{t}$ Abfall [5] steigern.

Entscheidend ist, dass dadurch im Vergleich zur Deponie eine Verminderung bei den Treibhausgasen in einer Größenordnung von ca. 700-1.300 $\mathrm{kg} \mathrm{CO}_{2} / \mathrm{t}$ Abfall erfolgen kann.

\subsection{Energie in industrieller Mitverbrennung}

Die Mitverbrennung in industriellen Feuerungsanlagen (=energetische Verwertung von Ersatzbrennstoffen) und die energetische Nutzung von Siedlungsabfällen in Müll-verbrennungsanlagen können schwer direkt verglichen werden. Beide Verfahren sind für den Einsatz unterschiedlicher Abfälle ausgelegt.

Industrielle Verbrennungsanlagen wie z. B. Zementwerke und Wirbelschichtanlagen benötigen aufbereitete Abfälle mit definierter Qualität (= Ersatzbrennstoff) und mittleren oder höheren Heizwerten. Gerade in diesem Fall erfolgt die direkte Substitution von

Tab. 1: Ergebnisse der $\mathbf{C O}_{2}$-Bilanz eines Ersatzbrennstoffes (EBS)

\begin{tabular}{lllll} 
Position & Ursprung & Wert & Dimension & entspricht \\
\hline Sammlung & & 0,01415 & $\mathrm{~kg} \mathrm{CO}_{2} / \mathrm{kg} \mathrm{HD}-\mathrm{EBS}$ & $1,31 \%$ \\
\hline Aufbereitung & 0,00198 & $\mathrm{~kg} \mathrm{CO}_{2} / \mathrm{kg} \mathrm{HD}-\mathrm{EBS}$ & $0,18 \%$ \\
\hline Transport & & 0,00950 & $\mathrm{~kg} \mathrm{CO}_{2} / \mathrm{kg} \mathrm{HD}-\mathrm{EBS}$ & $0,88 \%$ \\
\hline Verwertung & biogen & 0,54800 & $\mathrm{~kg} \mathrm{CO}_{2} / \mathrm{kg} \mathrm{HD}-\mathrm{EBS}$ & $50,57 \%$ \\
\hline & fossil & 0,51000 & $\mathrm{~kg} \mathrm{CO}_{2} / \mathrm{kg} \mathrm{HD}-\mathrm{EBS}$ & $47,06 \%$ \\
\hline Summe & & 1,08363 & $\mathrm{~kg} \mathrm{CO}_{2} / \mathrm{kg} \mathrm{HD}-\mathrm{EBS}$ & $100,00 \%$ \\
\hline
\end{tabular}

fossilen Energieträgern durch Ersatzbrennstoffe mit biogenen Anteilen. Hohe Wirkungsgrade in den Verbrennungsanlagen führen bei der Bilanzierung von Treibhausgasen $\mathrm{zu}$ hohen Gutschriften. Besonders gute Übersicht über die Beiträge der industriellen Mitverbrennungsanlagen zum österreichischen Energiehaushalt und Klimaschutz geben die Arbeiten von Hackl und Mauschitz.

Das deutsche UBA gibt die durchschnittliche Gutschrift für Mitverbrennung von Ersatzbrennstoffen (Zementwerk und Kraftwerk) mit $1.006 \mathrm{~kg} \mathrm{CO}_{2} / \mathrm{t} \mathrm{Ab}$ fall an. [5]

Im Rahmen eines Forschungsprojektes der SDAG wurden die $\mathrm{CO}_{2}$ Emissionen eines mittelkalorischen Ersatzbrennstoffes bilanziert [6]. Die Bilanz umfasste Sammlung, Ersatz-brennstoff Produktion, Transport zum und energetische Verwertung im Zementwerk. Die Feststellung des biogenen Anteils erfolgte mittels C14 Methode. Die $\mathrm{CO}_{2}$-Emission von Sammlung, Herstellung des Ersatzbrennstoffes und Transport zum Verwerter ist mit nur 2,37 \% der Gesamtemission gering und im Vergleich zu den Emissionen bei der Verwertung zu vernachlässigen (Tabelle 1). Auffällig ist weiters, dass knapp über $50 \%$ der gesamten $\mathrm{CO}_{2}$-Emissionen biogenen Ursprungs sind. Abbildung 6 zeigt das Ergebnis der Bilanz in Form eines Sankey-Diagrammes.

\subsection{Getrennte Sammlung und getrennte mechanische Behandlung von kommu- nalen und gewerblichen Siedlungsabfällen}

Praktische Erfahrungen und Untersuchungen bestätigen den Sinn der getrennten Behandlung von „Siedlungsabfällen aus Gewerbe" und „kommunalen Siedlungsabfällen“. Die gemeinsame Behandlung und somit Vermischung von „kommunalen Siedlungsabfällen“ (feucht, energiearm, hoher biogener Anteil) mit „Siedlungsabfällen aus Gewerbe" (trocken, energiereich, geringer biogener Anteil) ist aus Sicht der Ressourcennutzung, der Energieeffizienz und abfallwirtschaftlicher Überlegungen unbedingt zu vermeiden. Die getrennte Sammlung und die getrennte Behandlung in spezialisierten Anlagen ist sinnvoll. [7]

- Bei getrennter Behandlung wird ein höherer Anteil an hochkalorischer Leicht-fraktion und mittelkalorischem EBS erreicht.

- Die Ausbeute an energetisch nutzbarem EBS ist bei getrennter Behandlung deutlich höher.

- Die zu beseitigende Menge auf der Deponie erhöht sich deutlich bei gemeinsamer Behandlung in der MBA.

- Die Energiebilanz zeigt, dass die getrennte Behandlung deutlich energieeffizienter ist als die gemeinsame Behandlung in der MBA. Der Energieinhalt in den energetisch nutzbaren Frak- 
Tab. 2: Bundesweites Abfallaufkommen stofflich verwerteter

Siedlungsabfälle [8], [9]

\begin{tabular}{ll}
\hline Abfallart & Menge 2006 in t \\
\hline Altpapier & 654.000 \\
\hline Altglas* $^{*}$ & 195.000 \\
\hline Altholz & 143.000 \\
\hline Leichtfraktion* & 129.000 \\
\hline Altmetalle und Haushaltsschrott & 117.000 \\
\hline Altmetalle & 41.813 \\
\hline "gemäß ArgeV und AGR & \\
\hline
\end{tabular}

Tab. 3: Treibhausgas- und Energiegutschriften einzelner

Abfallarten [5]

\begin{tabular}{lcc}
\hline Abfallart & $\mathbf{~} \mathbf{g ~ \mathbf { C O } _ { 2 }}$ /t Abfall & $\mathbf{G J} / \mathbf{t}$ \\
\hline Altpapier & -217 & $-3,23$ \\
\hline Altglas & -192 & $-2,01$ \\
\hline Altholz & -304 & $-4,06$ \\
\hline Leichtfraktion & -299 & $-7,11$ \\
\hline Altmetalle und Haushaltsschrott & -3.094 & $-31,67$ \\
\hline Altmetalle & -3.094 & $-31,67$ \\
\hline
\end{tabular}

tionen (Leichtfraktion + EBS ) ist bei getrennter Behandlung deutlich hoher als bei gemeinsamer Verarbeitung .

Aus energetischer Sicht steht der Vorteil der getrennten Sammlung und Behandlung von Gewerbeabfällen außer Zweifel. Ist auch aus Klimaschutzgründen diese Trennung sinnvoll? Diese Frage ist jedenfalls zu bejahen. Das höhere Ausbringen an energetisch nutzbarem Ersatzbrennstoff und der höhere gewinnbare Energieinhalt führen zu positiven Treibhausgaseffekten. Aus einer Simulationsrechnung [7] lässt sich ableiten, dass durch gemeinsame Behandlung von Gewerbeabfällen in der MBA eine zusätzliche $\mathrm{CO}_{2}$ Belastung von $260 \mathrm{~kg} \mathrm{CO}_{2} / \mathrm{t}$ Gewerbeabfall zu veranschlagen ist. Anders ausgedrückt durch die getrennte Sammlung und Behandlung von Gewerbeabfällen in spezialisierten Splittinganlagen können im Ver- gleich zur Behandlung dieser Abfälle in der MBA pro t Gewerbeabfall $260 \mathrm{~kg} \mathrm{CO}_{2}$ vermieden werden.

Anschlusszwänge, die gut aufbereitbare Gewerbeabfälle zwanghaft einer MBA zuführen, sind daher nicht nur aus energetischer Sicht, sondern auch aus Sicht des Klimaschutzes strikt abzulehnen.

\subsection{Stoffliche Verwertung}

Abfälle werden verstärkt getrennt erfasst und stofflich verwertet. Grundsätzlich bedeutet das ein höheres Maß an Rohstoffschonung und verminderten Energieeinsatz im Vergleich zur Nutzung von Primärrohstoffen. Ein hoher Anteil der österreichischen Siedlungsabfälle - vor allem Altpapier, Altglas, Verpackungen, Altmetalle und Bioabfall - gehen in die Verwertung. Natürlich befindet sich der Anteil an Abfällen, die einem stofflichen Recycling zugeführt werden, bereits seit langem auf hohem Niveau.

Meist wird übersehen, dass auch jene Mengen die stofflich verwertet werden, einen positiven Beitrag zur Treibhausgasreduktion leisten. Das UBA Deutschland [5] gibt für Abfälle zur stofflichen Verwertung folgende Treibhausgasgutschriften aus:

Das stoffliche Recycling von Abfällen ermöglicht Energieeinsparungen; diese sind für die Treibhausgasvermeidung hauptverantwortlich. Besonders deutlich ist der Vorteil der stofflichen Verwertung bei Metallen, z. B. Metallschrotte die in Abfallbehandlungsanlagen mit Magnetabscheidern (Fe) oder Wirbelstromscheidern (NE) gewonnen werden.

Bei der Stahlproduktion auf Basis von Schrott anstelle von Roheisen beträgt die $\mathrm{CO}_{2}$-Einsparung $86 \%$. Für jede Tonne Stahlschrott brauchen 1,5 t Erz nicht abgebaut und eingeschmolzen, 0,5 t Brennstoff (Kohle, Öl) nicht gefördert und im Hochofen eingesetzt werden. Die Energieeinsparung beträgt ca. $90 \%$. Pro Tonne Eisen aus Abfall werden in der Primärproduktion $2.400 \mathrm{~kg} \mathrm{CO}_{2}$ vermieden. [10]

Pro Tonne Aluminium aus Abfall werden in der Primärproduktion $10.060 \mathrm{~kg} \quad \mathrm{CO}_{2}$ vermieden. $95 \%$ der Energie werden eingespart. Pro Tonne Kupfer aus Abfall werden in der Primärproduktion $5.530 \mathrm{~kg} \mathrm{CO}$ vermieden. [4]

Konsequente Metallabscheidung und Rückführung in den Stoffkreislauf bringt trotz relativ geringen Anteils an Abfallgemischen hohe $\mathrm{CO}_{2}$-Einsparungen. Getrennte Sammlung, optimierte Abtrennung in mechanischen Abfallbehandlungsanlagen und leistungsfähige Schlackenaufbereitungen sind in Bezug auf Treibhausgasvermeidung sehr effektiv.

Für Deutschland liegt eine umfassende Studie [5] für Siedlungsabfälle (Haus- und Sperrmüll, 


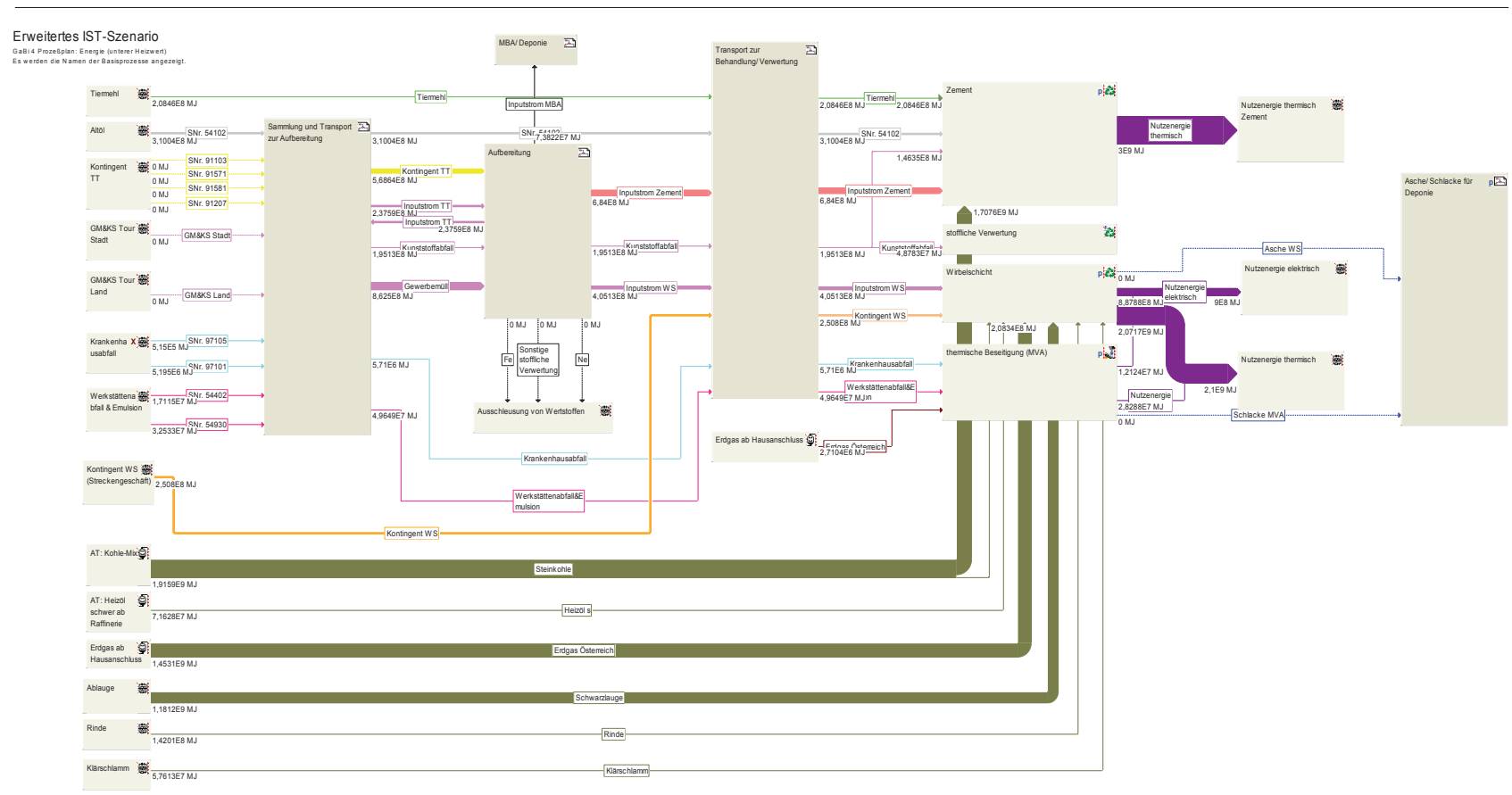

Abb. 7: Modell Energie- und Klimabilanz der SDAG [11]

hausmüllähnliche Gewerbeabfälle, Wertstoffe in Summe 40,9 Mio. Tonnen für 2005) vor. Insgesamt sind die gesamten $\mathrm{CO}_{2}$-Emissionen (Belastungen) geringer als die Gutschriften für die substituierten Prozesse. Die Bilanz weist also Nettoeinsparungen für die Sammlung und Behandlung von Siedlungsabfällen auf. Die nachgewiesene Treibhausgaseinsparung ist $2005 \mathrm{zu} 47 \%$ auf die stoffliche Verwertung von Altpapier, Altglas, Altholz/Sperrmüll, Metallen und Kunststoffen/LVP zurückzuführen.

Der positive Beitrag der stofflichen Verwertung zur positiven Treibhausgasbilanz in der Abfallwirtschaft ist offensichtlich hoch. Allerdings ist ein weiteres Verbesserungspotential nur geringfügig gegeben, da bereits hohe Sammel- und Verwertungsquoten bestehen.

Der Beitrag der stofflichen Verwertung ist für Österreich ähnlich einzuschätzen. Allein bei Bewertung der in Tabelle 2 dargestellten getrennt gesammelten Siedlungsabfälle mit den Daten aus
Tabelle 3, lässt sich ein positiver Beitrag zur Treibhausgasreduktion in der Höhe von ca. $753.000 \mathrm{t}$ $\mathrm{CO}_{2}$ äq und eine Energieeinsparung von ca. 9.032.000 GJ (entspricht ca. 220.000 t Heizöl). Bei Berücksichtigung aller Abfallströme in stoffliche Verwertung in Österreich sollte ein noch deutlich höherer Beitrag der österreichischen Abfallwirtschaft zum Klimaschutz darstellbar sein. Die genaue und zusammenfassende Untersuchung dieses Beitrages der stofflichen Verwertung zum Klimaschutz wäre aus Sicht des Autors sinnvoll.

Jedenfalls ist der positive Beitrag der stofflichen Verwertung bisher in der Diskussion noch nicht ausreichend dargestellt worden.

Der Ausbau der getrennten Sammlung, die verstärkte getrennte Erfassung von Wertstoffen - insbesondere in Industrie und Gewerbe - und generell die Erhöhung der Mengen in die stoffliche Verwertung - insbesondere der Metallfraktionen - ist ein positiver Beitrag der Abfallwirtschaft und insbesondere von Abfallsammlern/Entsorgungsunternehmen zum Klimaschutz.

\subsection{Joint Implementation (JI) und Clean Developement Mechanism (CDM)}

Die Projekt bezogenen Mechanismen Joint Implementation (JI) und Clean Development Mechanism (CDM) sind im Kyotoprotokoll festgelegte Instrumente. Sie ermöglichen es den Industrieländern einen Teil ihrer Verpflichtungen zur Minderung ihrer Treibhaus-gasemissionen auch außerhalb ihres Territoriums einzulösen.

Maßnahmen zur Treibhausgasreduktion in anderen Ländern können im Rahmen des EU-Emissionshandels angerechnet werden.

Besondere Bedeutung für die Abfallwirtschaft haben Deponieprojekte. Einige österreichische Entsorgungsunternehmen sind hier bereits seit einigen Jahren erfolgreich tätig und setzen JI- bzw. CDM-Projekte um. 


\section{Unternehmensspezifische Beiträge zum Klimaschutz}

\subsection{Energie- und Klimabilanz eines Entsorgungsunter- nehmens}

Ziel der Energie- und Klimabilanz der Saubermacher Dienstleistungs AG (SDAG) war die Darstellung des Klimaschutzbeitrages eines Entsorgungsunternehmens unter Einbeziehung der Behandlungswege relevanter Abfallströme und der Vergleich mit Alternativszenarien Zentraler Bestandteil der Studie [11] war die Erstellung einer Stoffflussanalyse für die thermisch behandelten Abfallströme. Die relevanten Prozesse sind Sammlung und Transport zur Aufbereitung, die Aufbereitung selbst, Transport zur Behandlung/Verwertung, die verschiedenen Anlagen der Behandlung und Verwertung, sowie MBA/Deponie und Asche/Schlacke für Deponie sowie Hilfsprozesse und alternative Energieträger (siehe Abbildung 7).

Ergebnisse der unternehmensspezifischen Energie und Klimabilanz:

- Einsparung von $153.000 \mathrm{t} \mathrm{CO}_{2}$ durch Abfallvorbehandlung und industrielle Mitverbrennung im Vergleich zur herkömmlichen Müllverbrennung ( jährlicher $\mathrm{CO}_{2}$ Ausstoß von 61.000 Pkws)

- Die $\mathrm{CO}_{2}$ Emissionen durch Logistik und Aufbereitung betragen nur $4 \%$ der gesamten $\mathrm{CO}_{2}$ Emissionen.

- Energieaufwand durch Logistik und Aufbereitung betragen nur 3,8\% der Nutzenergie der Abfälle.

In die Bilanz wurden bewusst die Behandlungs- und Verwertungsanlagen miteinbezogen, obwohl diese meist nicht durch SDAG selbst betrieben werden. Die Entscheidung welchem Behandlungsweg ein Abfall zugeführt wird, ist aber eine Entscheidung des Entsorgungsunternehmens. Eine aussagekräftige Treibhausgasbilanz muss die gesamte Prozesskette von der Sammlung, über die mechanische Behandlung bis zur thermischen Behandlung umfassen. In einem weiteren Schritt sollten auch Abfallströme, die einer stofflichen Verwertung zugeführt werden, in der Bilanz berücksichtigt werden.

Die Energie und Klimabilanz eines Entsorgungsunternehmens sollte als Beitrag zur Darstellung der „Qualität“ von Entsorgungsdienstleistungen eines Entsorgungsunternehmens gesehen werden.

\subsection{Einsparung von $\mathrm{CO}_{2}$ auf- grund Optimierungen der Logistik}

Abfallentsorger sind vielfach Logistik dominierte Unternehmen. Die Abfallsammlung und der Transport zu den Behandlungsanlagen erfolgt meist durch LKWs. Die spezifische $\mathrm{CO}_{2}$ Emission pro transportierter $\mathrm{t}$ Abfall kann durch folgende Maßnahmen reduziert werden:

- Nutzung regionaler Behandlungsanlagen

- Reduktion von Leerfahrten

- Nutzung von Transportsystemen mit hoher Nutzlast

- Optimale Nutzung der zulässigen Nutzlast

- Nutzung von Bahntransporten bei hohen Transportdistanzen

- Optimierung von Sammeltouren

- Routenoptimierung durch GPS Lösungen

Folgendes praktisches Beispiel zeigt die Größenordnung einer $\mathrm{CO}_{2}$ Reduktion durch Logistikoptimierung: Durch die Belieferung einer näher gelegenen Ersatzbrennstoff Verwertungsanlage (neue Anlage liegt $139 \mathrm{~km}$ näher) konnten nicht nur Transportkosten gesenkt, sondern auch der $\mathrm{CO}_{2}$-Ausstoß um $13 \mathrm{~kg} / \mathrm{t}$ reduziert werden. Aufgrund der Reduktion der Transportkilo- meter ergibt sich bei einer jährlichen Abfallmenge von $40.000 \mathrm{t}$ eine Einsparung von 520 t $\mathrm{CO}_{2} / \mathrm{a}$.

Wenn man die positiven $\mathrm{CO}_{2}$ Effekte der Logistikoptimierung mit den positiven Effekten der thermischen oder stofflichen Verwertung vergleicht, ist festzustellen, dass die Effekte der Verwertung bei weitem überwiegen.

\subsection{Einsatz emissionsarmer Fahrzeuge}

Die Saubermacher Dienstleistungs AG stellt Ende 2007 in Wien das erste Abfallsammel-fahrzeug mit Erdgas-Antrieb in den Dienst. Der 26-Tonner verfügt über $205 \mathrm{~kW} / 279$ PS und ein maximales Drehmoment von 1000 Nm. Das Fahrzeug ist für kurze Distanzen im städtischen Bereich optimal. Eine Tankfüllung von 140 Kilogramm Erdgas (55 MJ/kg) reicht für die Tagesstrecke eines Abfallsammelfahrzeugs. Der Verbrauch dieser LKW liegt nach bisherigen Erfahrungen bei ca. $40 \mathrm{~kg}$ Erdgas auf $100 \mathrm{~km}$ im Müllsammelverkehr (Stop-and-go Betrieb).

Ein umfassender Emissionsvergleich des Schweizer Forschungsinstituts EMPA Materials Science and Technology ergab deutliche Vorteile für Fahrzeuge mit Erdgasantrieb gegenüber solchen mit Benzin- oder Dieselmotoren. Eines der zentralen Ergebnisse: Erdgasfahrzeuge produzieren circa 21 Prozent weniger Treibhausgase als Benzinfahrzeuge und ca. 11 Prozent weniger als Dieselfahrzeuge. [12]

Eine Untersuchung des ÖAMTC, der TU-Wien, General Motors und OMV ergab, dass der Erdgas-Motor bis zu zehn Prozent weniger $\mathrm{CO}_{2}$ abgibt, zu 85 Prozent weniger Partikel als der Diesel (ohne Partikelfilter) und zu einem kaum nachweisbaren Niveau Stickstoffoxide in die Luft abgibt. Bei dieser Untersuchung war allerdings ein monovalenter Erdgas- 
Motor angetreten, also ein rein mit Erdgas betriebenes Fahrzeug. [13] Die Lärmemissionswerte liegen bis zu 50 Prozent unter jenen eines Dieselmotors.

\section{5. „Richtiger Abfall in richtige Anlage"}

Die konsequente Trennung von Gewerbeabfällen (Siedlungsabfall aus gewerblicher Sammlung) und Hausmüll (Siedlungsabfall aus kommunaler Sammlung) in der Sammlung und bei der mechanischen Behandlung ermöglicht höhere Energieeffizienz und $\mathrm{CO}_{2}$ Einsparungen. Insbesondere für die gemeinsame Sammlung von Hausmüll und Gewerbeabfall wurde nachgewiesen, dass die Trennbarkeit in der MBA und damit der Anteil der thermisch verwertbaren Fraktionen und ihre Qualität (Heizwert) negativ beeinflusst werden. [7]

Splittinganlagen sind auf trockene, gut aufbereitbare Gewerbeabfälle mit geringem biogenen Anteilen ausgerichtet und erreichen ein sehr hohes Ausbringen an energetisch verwertbaren Fraktionen. MBA ist ein Verfahren zur Vorbehandlung vor der Deponierung und ist auf feuchte Siedlungsabfälle mit hohem biogenen Anteil spezialisiert. Der geringere Anteil an thermisch verwertbaren Fraktionen erreicht daher energetisch tendenziell niedrigere Qualität.

DurchkonsequenteZuordnung von Abfällen zur geeigneten Anlage (Splittinganlage, MBA, Sortieranlage, EBS-Produktionsanlage, MVA) kann der jeweilige Abfall mit höchstmöglichem Nutzen behandelt werden. Rein ökonomisch begründete Stoffstromlenkung z. B. durch „Anschlusszwang“ verhindern die Zuordnung zur sinnvollsten Anlage und sind daher aus Sicht des Klimaschutzes und der Energieeffizienz abzulehnen. [7]

Waste Management in Europe

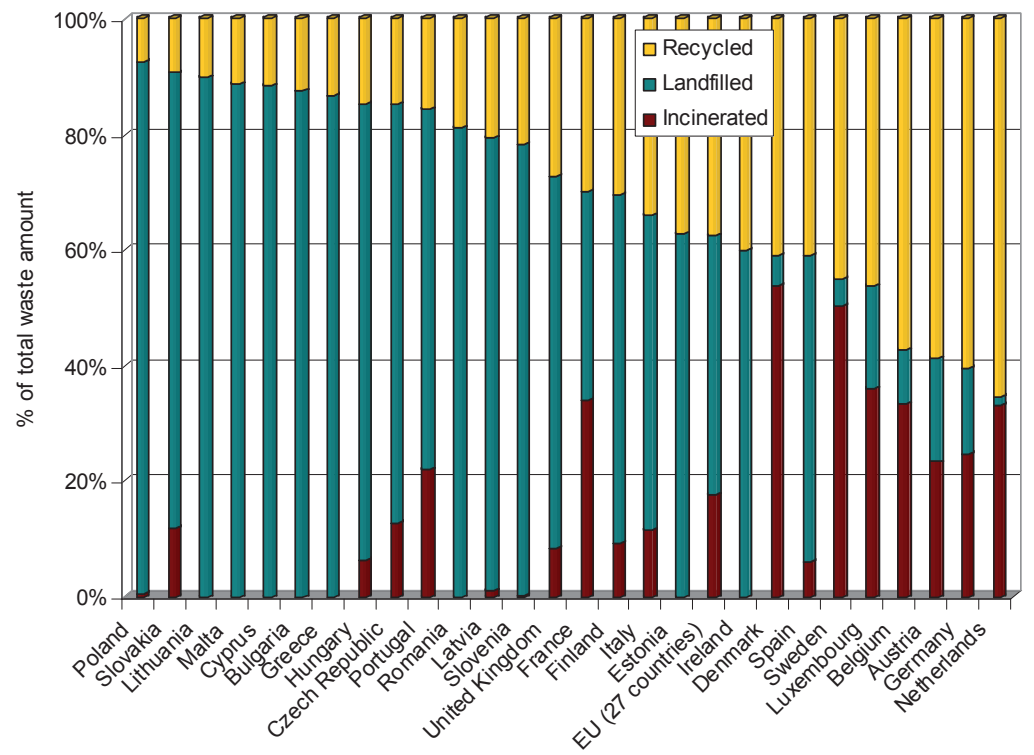

Abb. 8: Abfallbehandlung EU27 2005 [14]

\subsection{Verstärkung der ge- trennten Sammlung ins- besondere in CEE}

Wahrend der Anteil der getrennten Sammlung und der stofflichen Verwertung von Abfällen in Österreich bereits sehr hoch ist, besteht in den Ländern Zentral- und Osteuropas (CEE) noch deutlicher Aufholbedarf. Vielfach ist die getrennte Sammlung noch nicht eingeführt. Abbildung 8 gibt einen Überblick über die Recyclingquoten in der Europäischen Union.

Durch die stoffliche Verwertung von getrennt gesammelten Abfällen können aber deutliche positive $\mathrm{CO}_{2}$ Effekte erreicht werden (vergl. Kap. 3.5 ). Die $\mathrm{CO}_{2}$ Emission der Sammlung und ggf. Behandlung von Abfällen ist im Vergleich zu den positiven Effekten bei der Verwertung $\mathrm{zu}$ vernachlässigen. Jede Tonne Altpapier, Altglas, Altholz, Metalle oder Kunststoffe die getrennt gesammelt und stofflich verwertet werden ist ein positiver Beitrag zur Treibhausgasreduktion. Während die Verbesserung der Sammel- und Verwertungsquoten in Österreich an seine Grenzen stößt, erschließt sich in den Ländern CEE großes Marktpotential. Leider ist der positive Beitrag der getrennten Sammlung und stofflichen Verwertung im Zusammenhang mit Klimaschutz noch nicht ausreichend bekannt.

Die SDAG ist wie andere österreichische Entsorgungsunternehmen in diesen Ländern wirtschaftlich aktiv. Der Transfer abfallwirtschaftlichen Knowhows und Aufbau des Sammlungs- und Verwertungsmarktes ist ein effektiver Beitrag österreichischer Entsorger zum Klimaschutz.

\section{Was trägt die euro- päische Abfallwirtschaft zum Klimaschutz bei?}

In der Europäischen Union (EU27, 2005) fielen ca. 254 Mio.t Siedlungsabfälle an , davon wurden ca. 95 Mio. t ( $37 \%)$ recycled, 44 Mio. $\mathrm{t}(\sim 18 \%)$ thermisch behandelt und 114 Mio. $t(\sim 45 \%)$ deponiert. [14]

Der Abfallsektor (Deponie, Verbrennung, Abwasserbehandlung) der EU15 konnte zwischen 1990 und 2003 die $\mathrm{CO}_{2}$-Emissionen um 44 Mio. t $\mathrm{CO}_{2}$ äq (ca. 40 \%) reduzie- 
ren. $90 \%$ der erreichten Reduktion sind auf im Abfallsektor eingesparte Methanemissionen von Deponien zurückzuführen.

Würden alle europäischen Staaten die Deponierung unbehandelter Abfälle zeitnah unterbinden, bestünde ein Reduktionspotential von ca. 110 Mio.t $\mathrm{CO}_{2}$ äq/a bezogen auf 1990. Gemessen an der geplanten europaweiten Reduktion (EU15) von 1.266 Mio. t $\mathrm{CO}_{2}$ äq ist der mögliche Anteil des Sektors Abfallwirtschaft bemerkenswert hoch (ca. 9 \%).

In vielen Ländern der $\mathrm{EU}$ ist der abfallwirtschaftliche Standard (Verbrennung, Deponierung, stoffliche Verwertung) noch nicht vergleichbar mit der Situation in Österreich oder Deutschland. Das deutsche Umweltbundesamt hat das Potential der europäischen $\mathrm{Ab}$ fallwirtschaft zur $\mathrm{CO}_{2}$-Reduktion abgeschätzt. Wenn man annimmt, dass Abfälle der EU15 ähnlich behandelt werden wie in Deutschland (Szenario 2020)

- Aufgabe der Deponierung von nicht vorbehandelten Abfällen

- getrennte Erfassung und Verwertung von Bioabfällen

- Ausbau der stofflichen Verwertung

- Ausbau der Mitverbrennung (Ersatzbrennstoffe)

- Bau von Verbrennungsanlagen nach modernstem Stand der

\section{Technik}

so lässt Sich eine Einsparung von 134 Mio. $\mathrm{CO}_{2}$ äq in Bezug auf das Jahr 2000 darstellen. Diese pro Jahr in der europäischen Abfallwirtschaft einsparbare Treibhausgasmenge liegt ca. $50 \%$ über der jahrlichen Gesamt-Treibhausgasemission Österreichs. Das unterstreicht das wesentliche Einsparungspotential des Sektors.

\section{Korrespondenzadresse:}

Prok. DI Dr. Roland Pomberge

Saubermacher Dienstleistungs AG

r.pomberger@saubermacher.at

\section{LITERATUR}

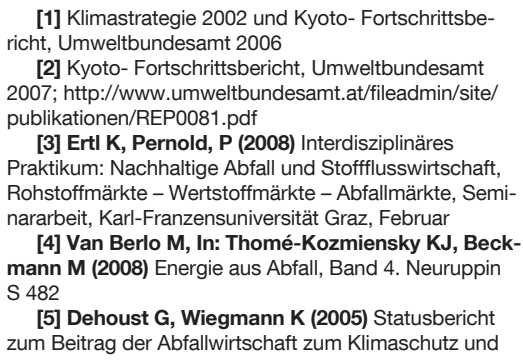

[1] Klimastrategie 2002 und Kyoto- Fortschrittsbericht, Umweltbundesamt 2006

[2] Kyoto- Fortschrittsbericht, Umweltbundesamt 2007; http://www.umweltbundesamt.at/fileadmin/site/ 2007; http://www.umweltbu

[3] Ertl K, Pernold, P (2008) Interdisziplinäres Praktikum: Nachhaltige Abfall und Stoffflusswirtschaft, Rohstoffmärkte - Wertstoffmärkte - Abfallmärkte, Seminararbeit, Karl-Franzensuniversität Graz, Februar [4] Van Berlo M, In: Thomé-Kozmiensky KJ, Beckmann M (2008) Energie aus Abfall, Band 4. Neuruppin $\mathrm{S} 482$

[5] Dehoust G, Wiegmann K (2005) Statusbericht zum Beitrag der Abfallwirtschaft zum Klimaschutz und

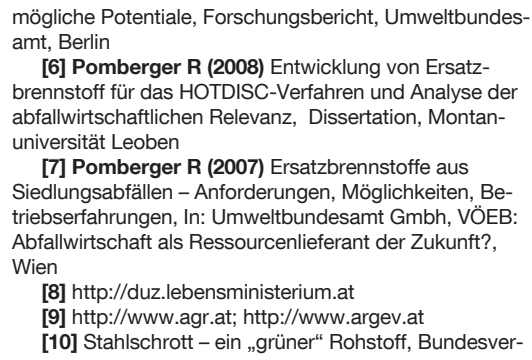

mögliche Potentiale, Forschungsbericht, Umweltbundesamt, Berlin

[6] Pomberger R (2008) Entwicklung von Ersatzbrennstoff für das HOTDISC-Verfahren und Analyse de abfallwirtschaftlichen Relevanz, Dissertation, Montanuniversität Leoben

[7] Pomberger R (2007) Ersatzbrennstoffe aus Siedlungsabfällen - Anforderungen, Möglichkeiten, BeSiedlungsabfällen - Anforderungen, Möglichkeiten, Betriebserfahrungen, In: Umweltbundesamt Gmbh, VOEB: Abfall

[8] http://duz.lebensministerium.at

[9] http://www.agr.at; http://www.argev.at

[10] Stahlschrott - ein „grüner“ Rohstoff, Bundesver-

einigung Deutscher Stahlrecycling- und Entsorgungsunternehmen e.v. (BDSV) www.bdsv.de

[11] Curtis A, Scherübl T, Ragoßnig A, Wartha C, Weber S (2007) Klimaschutzbeitrag der Saubermacher Dienstleistungs AG Update 2006, Fachhochschulstudiengänge Burgenland $\mathrm{GmbH}$

[12] http://www.pressemeldungen.at/24171/neuestudie-belegt-sauberkeit-von-erdgasautos/

[13] http://www.oeamtc.at/index.php?type=article\&id $=1120793$ \&menu_active $=0311$

[14] Sander K (2008) Climate protection potentials of eu recycling targets, Ökopol GmbH 